\title{
Monte Carlo calculations with nuclear point data based on JEF-2.2 and JEFF-3.1 for the VENUS-7 critical benchmarks
}

\author{
W. Zwermann and S. Langenbuch \\ Gesellschaft fuer Anlagen- und Reaktorsicherheit (GRS) mbH, Forschungsinstitute, 85748 Garching, Germany
}

\begin{abstract}
Currently, the VENUS-7 series of critical experiments is evaluated within an OECD/NEA benchmark activity for the validation of nuclear data and codes for MOX fuel. Measurements are available for various cold square lattice cores, consisting of an inner MOX and an outer $\mathrm{UO}_{2}$ zone, moderated and reflected by light water. In addition to the multiplication factors, reactivity changes for substitution or removal of a small number of fuel pins, as well as radial pin-wise fission rate distributions are known from the experiments. Results obtained with the MCNP code, using point-wise cross section libraries processed from the JEF-2.2 and JEFF-3.1 evaluated data, are presented. Concerning the reactivity differences and fission rate distributions, satisfactory agreement between the calculated and measured results is obtained. The absolute values of the multiplication constants are underestimated with the JEF-2.2 data by approximately $700 \mathrm{pcm}$, which is consistent with the results of earlier calculations for the KRITZ-2 LWR lattice $\mathrm{UO}_{2}$ cores. When using the new JEFF-3.1 data available from the NEA data bank, the calculated multiplication constants increase by a statistically significant amount of about $200 \mathrm{pcm}$. The results supplement a series of benchmark calculations with the Monte Carlo method on critical experiments performed for the validation of evaluated nuclear point data libraries.
\end{abstract}

\section{Introduction}

For the neutronic description of complex systems, the Monte Carlo method with continuous nuclear data libraries is particularly suited. Using Monte Carlo codes, on one hand, there are no substantial restrictions in the geometrical modelling of the arrangements under consideration. Point-wise cross section data can be used directly without the necessity of a preceding resonance treatment, as it is the case when using codes with multi-group representation of the cross sections. Hence, the Monte Carlo method can be used for directly validating nuclear data libraries. On the other hand, the performance of computers available today is high enough to reduce the statistical uncertainties of Monte Carlo calculations to such a degree that sufficiently precise results can be generated even for local quantities like radial or even axial pin power distributions, or small reactivity differences between similar states of a system.

Over the past few years, we have undertaken extensive benchmark studies for criticality calculations on a broad spectrum of fissile systems with the Monte Carlo code MCNP [1] using different nuclear data libraries [2,3]. With the JEF-2.2 based data we use routinely for water moderated systems, it turned out that the multiplication factors of LEU LWR type lattices tend to be slightly underestimated. In the present paper, we report in detail on our recent benchmark calculations of the VENUS-7 MOX/UO $\mathrm{UO}_{2}$ critical assemblies, which are currently being evaluated within an OECD/NEA benchmark activity. The calculations were performed with the above-mentioned JEF-2.2 data, and a JEFF-3.1 library which is available from the NEA data bank. The calculations will be discussed with special attention to the differences between the results obtained with JEF-2.2 and JEFF-3.1. The results will be compared with the results of re-calculations of the KRITZ-2 critical assemblies with the same nuclear data libraries.

\section{The VENUS-7 benchmark}

The VENUS-7 [4] series of critical experiments is currently evaluated within an OECD/NEA benchmark activity for the validation of nuclear data and codes for MOX fuel. The VENUS-7 cores are cold square lattices containing approximately 900 fuel pins each. The assemblies differ in their radial shapes; the configurations $7 / 0$ and $7 / 1$ are approximately cylindrical, while $7 / 3$ is square-shaped. They consist of an inner MOX and an outer $\mathrm{UO}_{2}$ radial zone, and are moderated and reflected by light water. The main measured quantities are multiplication factors, reactivity differences caused by substitution or removal of fuel pins, and radial pin-wise fission rate distributions for selected pins. Sketches of the arrangements of fuel pins are given in figure 1 .

The active height of the fuel pins is $50 \mathrm{~cm}$, and the pitch is $1.303 \mathrm{~cm}$. The moderator/fuel ratio is 1.52 and 1.24 for the $\mathrm{UO}_{2}$ and MOX zones, respectively. The $\mathrm{UO}_{2}$ fuel has a $\mathrm{U} 235$ enrichment of $4 \%$, and the MOX fuel is a mixture of $\mathrm{UO}_{2}$ with an enrichment of $3 \%$ and $1 \%$ fissile plutonium. In addition to the basic states, some modified states were considered,
$7 / 0$

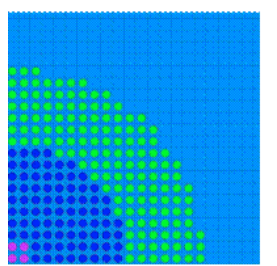

$7 / 1$

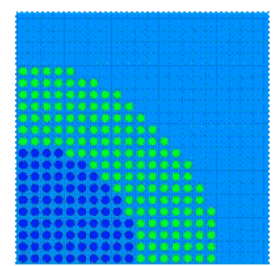

$7 / 3$

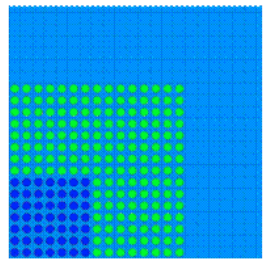

Fig. 1. Horizontal cut through one quarter of the VENUS-7 assemblies. Blue pins: MOX fuel; green pins: UO2 fuel. In assembly 7/0, the central pins for the substitution experiments are displayed in magenta. 
consisting of substituted fuel in the centre of assembly $7 / 0$, and the removal of a peripheral pin from assembly $7 / 1$. The substituted fuel was:

(1) the same $\mathrm{UO}_{2}$ as used for the outer core zone,

(2) MOX of the same composition as for the basic state, but with other radial dimensions,

(3) MOX with altered U-235 enrichment and fissile Pu content $(2 / 2.7 \%)$.

\section{Calculation methods}

Calculations were performed for the basic and modified assemblies with the Monte Carlo code MCNP-4C using different nuclear point data libraries. These are a set of data mainly based on JEF-2.2, generated by IKE Stuttgart and extensively validated in series of benchmark calculations performed in collaboration between IKE and GRS (see, e.g., ref. [2]), and a set of JEFF-3.1 based data available from the NEA data bank [5]. The $\mathrm{S}(\alpha, \beta)$ matrices were also taken from these distributions; for Plexiglas, which is present in some structure materials like grids and pin stops, polyethylene $\mathrm{S}(\alpha, \beta)$ data were used.

The assemblies were calculated in full three-dimensional $1 / 4$-core representation, modelling in the most possible detail which is known from the documentation of the experiments. 200 millions of neutron histories (20,000 active cycles with 10,000 neutrons each, and 100 discarded cycles for converging the source distribution) were evaluated, leading to statistical uncertainties as low as $5.0 \cdot 10^{-5}$ in the multiplication factors and $1.0 \cdot 10^{-3}$ in the axially averaged pin fission rates on the $1 \sigma$ level.

\section{Results}

First, the results for the differences for the multiplication factors due to fuel pin substitution or removal, for which experimental values exist, are presented in table 1 along with the measurements.

The agreement between corresponding values obtained with the different nuclear data sets is very satisfactory, with differences compatible with the statistical uncertainties. The agreement between measured and calculated values is somewhat poorer, with the correct signs and magnitudes of the calculated reactivity worths, but values which are slightly outside the intervals given by the experimental errors and the statistical uncertainties in the calculations. For the pin removal measurement in assembly $7 / 1$, the measured value was obtained by removing four pins (one pin per quarter-core), and correspondingly, the calculations were performed by removing the outmost pin in the diagonal direction. However, the positions of the pins removed in the experiment are not given in the documentation, introducing an extra uncertainty of the measured value.

In figure 2, the radial pin-by-pin fission rate distribution in the MOX zone of the VENUS-7/1 assembly in the direction along the $\mathrm{x}$ axis and in diagonal direction is displayed. The
Table 1. Differences in the multiplication factors in $\%$ for the VENUS-7/0 fuel pin substitutions and the VENUS-7/1 fuel pin removal, calculated with MCNP and two variants of JEF nuclear data libraries, in comparison with measured values. The uncertainties given for the MCNP calculations are $2 \sigma$.

\begin{tabular}{lccc}
\hline & Experiment & JEF- 2.2 & JEFF-3.1 \\
\hline $7 / 0$, subst.(1) & $+0.094 \pm 0.004$ & $+0.125 \pm 0.01$ & $+0.129 \pm 0.01$ \\
$7 / 0$, subst.(2) & $-0.058 \pm 0.004$ & $-0.090 \pm 0.01$ & $-0.073 \pm 0.01$ \\
$7 / 0$, subst.(3) & $-0.092 \pm 0.004$ & $-0.075 \pm 0.01$ & $-0.064 \pm 0.01$ \\
$7 / 1$, pin rem. & $-0.016 \pm 0.003$ & $-0.015 \pm 0.003$ & $-0.012 \pm 0.003$ \\
\hline
\end{tabular}

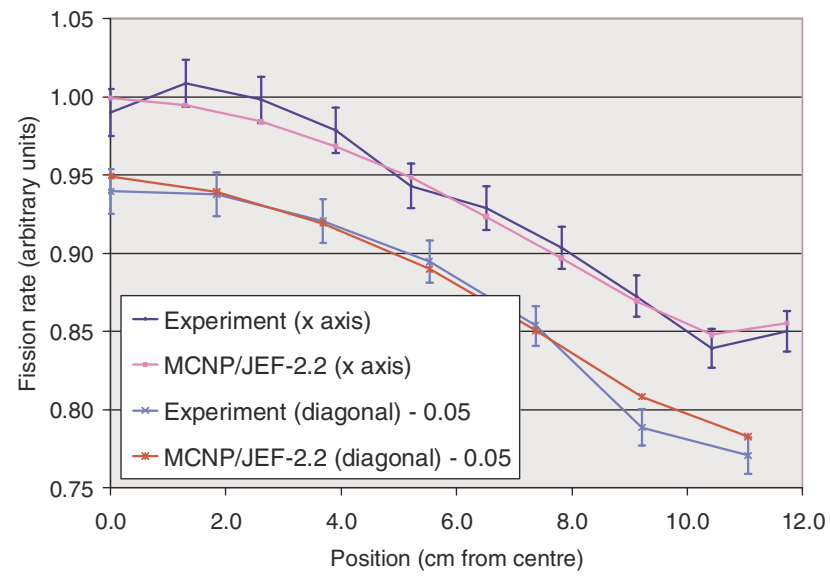

Fig. 2. Radial fission rate distributions in the MOX zone of the VENUS-7/1 core along different directions; MCNP results in comparison with measured values.

MCNP results obtained with the JEF-2.2 based nuclear data are compared with the measured values. The error bars plotted for the experimental points correspond to the value of $1.5 \%$ given in the specification. The experimental points are the average of the measured values of two fuel pins opposite to each other with respect to the centre of the core. The positions of the pins considered for the comparison are indicated in blue colour in figure 3, where the radial pin-by-pin fission rate calculated with MCNP is displayed.

There is satisfactory agreement between the calculated and the measured values with differences essentially within the uncertainties in the measurements; the statistical uncertainties in the MCNP results are not visible in figure 2 because of their small size of $\leq 0.1 \%$. When comparing the results obtained with JEF-2.2 and JEFF-3.1 nuclear data, the curves are not distinguishable in the representation of figure 2.

Table 2. Multiplication factors for the VENUS-7 assemblies, calculated with MCNP and two variants of JEF nuclear data libraries, in comparison with the measured values.

\begin{tabular}{lcccc}
\hline & Experiment & JEF-2.2 & JEFF-3.1 & Difference \\
\hline VENUS-7/0 & $\mathrm{n} / \mathrm{a}$ & 0.99248 & 0.99497 & 0.00249 \\
VENUS-7/1 & 1.0009 & 0.99354 & 0.99606 & 0.00252 \\
VENUS-7/3 & 0.9985 & 0.99300 & 0.99518 & 0.00218 \\
\hline
\end{tabular}




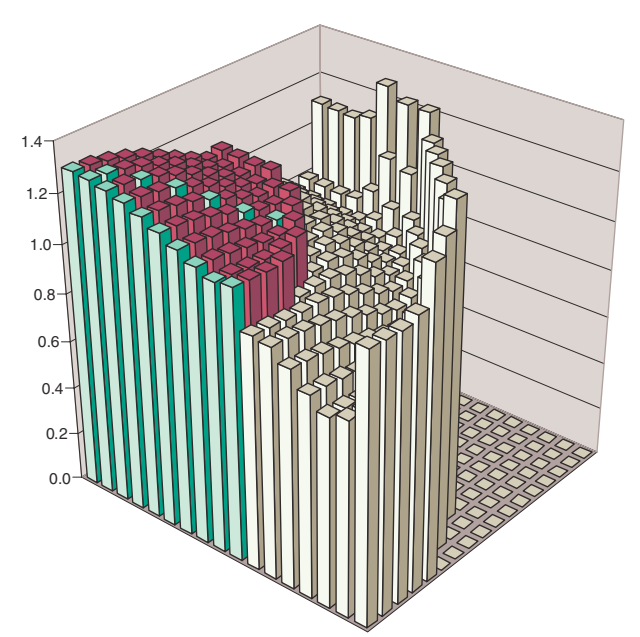

Fig. 3. Radial fission rate distribution for one quarter of the VENUS$7 / 1$ configuration calculated with MCNP. Pin positions where experimental values are available are marked in blue.

The multiplication factors for the basic configurations of the VENUS-7 assemblies are given in table 2. There is no measured value for assembly 7/0, but obviously the value should also be very close to 1.0 .

With the JEF-2.2 nuclear data, the experimental values of the multiplication factors of approximately 1.0 are significantly underestimated by about $700 \mathrm{pcm}$. This is consistent with the results of earlier MCNP calculations for the KRITZ-2 LWR lattice $\mathrm{UO}_{2}$ critical assemblies [6]. With the newer JEFF3.1 evaluation, the calculated multiplication factors increase by a statistically significant amount of more than $200 \mathrm{pcm}$. Thus we obtain a significantly better agreement of the calculated and measured multiplication factors; the differences are $400-500 \mathrm{pcm}$ in this case. It should be noted that an underestimation is observed in all solutions contributed to the benchmark so far.

This rise in the multiplication factors when going from JEF-2.2 to JEFF-3.1 is also qualitatively consistent with the results of calculations for the KRITZ-2 assemblies; the values are given in table 3 . The configurations $2: 1$ and 2:13 are $\mathrm{UO}_{2}$ lattices, whereas 2:19 is a MOX lattice. The experimental values are not explicitly stated; they are not given in the documentation of the measurements, so the assemblies are assumed to be in the critical state. The calculations were performed with 100 millions of neutron histories, resulting in statistical uncertainties of $\sigma=7.0 \cdot 10^{-5}$. For the cold KRITZ2 LEU configurations $2: 1$ and $2: 13$, the values of the reactivity differences when going from JEF-2.2 to JEFF-3.1 data are comparable to those found for the VENUS-7 assemblies. For the hot states of these configurations (at about $230{ }^{\circ} \mathrm{C}$ ), the differences are smaller. For the MOX core KRITZ-2:19, the differences are substantially larger (about $500 \mathrm{pcm}$ ), which even leads to a slight overestimation of the multiplication factors.

\section{Conclusions}

In summary, from the criticality calculations of the VENUS-7 assemblies with the Monte Carlo code MCNP using different
Table 3. Multiplication factors for the KRITZ-2 assemblies, calculated with MCNP and two variants of JEF nuclear data libraries.

\begin{tabular}{llll}
\hline & JEF-2.2 & JEFF-3.1 & Difference \\
\hline KRITZ-2:1 cold & 0.99396 & 0.99587 & 0.00191 \\
KRITZ-2:1 hot & 0.99299 & 0.99343 & 0.00044 \\
KRITZ-2:13 cold & 0.99649 & 0.99879 & 0.00230 \\
KRITZ-2:13 hot & 0.99571 & 0.99646 & 0.00075 \\
KRITZ-2:19 cold & 0.99746 & 1.00237 & 0.00491 \\
KRITZ-2:19 hot & 0.99646 & 1.00192 & 0.00546 \\
\hline
\end{tabular}

nuclear point data, we found a slight underestimation for the multiplication constants when using our standard JEF-2.2 library. When performing the calculations with the recently available JEFF-3.1 data, the differences between calculated and measured results is reduced, with a remaining underestimation of $400-500 \mathrm{pcm}$. A re-calculation of the KRITZ-2 benchmarks yields similar results for the LEU configurations. For reactivity differences caused by fuel pin substitutions or removal, as well as for radial pin-by-pin fission rate distributions, both libraries yield the same results within the statistical uncertainty intervals. The results are mostly compatible with the experimental values with quantitative differences for the reactivity worths for some cases, which are outside the experimental errors and the statistical uncertainties of the Monte Carlo calculations. Overall, the presented results confirm that the Monte Carlo method with up-to-date nuclear point data can be used to reliably describe the neutron physical parameters of LWR lattice systems. The trend for a slight underestimation of the multiplication constants of LEU pin lattice systems observed in calculations with JEF-2.2 based libraries is reduced when using the new JEFF-3.1 data.

This work is supported by the German Federal Ministry of Economics and Technology.

\section{References}

1. J.F. Briesmeister (ed.), MCNP - A General Monte Carlo NParticle Transport Code - Version 4C, LA-13709-M (2000).

2. W. Bernnat, M. Mattes, S. Langenbuch, W. Zwermann, Validation of Nuclear Data Libraries for Reactor Safety and Design Calculations, in Proceedings of the International Conference on Nuclear Data for Science and Technology, Tsukuba, Japan, 2001, p. 884.

3. W. Bernnat, M. Mattes, S. Langenbuch, W. Zwermann, Monte Carlo Reactor Physics Calculations for Critical Assemblies and LWR Full Core Models, Proceedings of the International Conference on the New Frontiers of Nuclear Technology (PHYSOR), Seoul, Korea, 2002, Paper No. 13A-03.

4. Benchmark on the VENUS Plutonium Recycling Experiments Configuration 7: Benchmark Specification, NEA/NSC/DOC (2005), p. 11.

5. Processing of the JEFF-3.1 Cross Section Library into a Continuous Energy Monte Carlo Radiation Transport and Criticality Data Library, NEA/NSC/DOC (2006) 18; http://www.nea.fr/abs/html/nea-1768.html

6. Benchmark on the KRITZ-2 LEU and MOX Critical Experiments: Final Report, NEA/NSC/DOC (2005), p. 24. 